\title{
Impact of diuretics on the urate lowering therapy in patients with gout: analysis of an inception cohort
}

\author{
Laura Ranieri ${ }^{1}$, Carolina Contero ${ }^{2}$, Maria-Luisa Peral ${ }^{1}$, Irene Calabuig ${ }^{1}$, Pedro Zapater ${ }^{2,3}$ and Mariano Andres ${ }^{1,2^{*}}$ (D)
}

\begin{abstract}
Background: Diuretics have been associated with impaired response and refractoriness in gout, but whether this effect is still present with new urate-lowering drugs (ULD) and treat-to-target strategies is unknown. The aim of the present study was to assess the impact of the diuretics on the response to ULD in patients with gout.

Methods: This was a retrospective analysis of an inception cohort. Participants were classified according to the type of ULD prescribed. We analysed the maximal dose of ULD (primary outcome variable), serum urate (SU) reduction, and the achievement of different SU targets $(6 \mathrm{mg} / \mathrm{dL}, 5 \mathrm{mg} / \mathrm{dL}$, and $4 \mathrm{mg} / \mathrm{dL}$ ), according to the type of ULD prescribed and use of diuretics (loop and/or thiazide). We adjusted for confounders using multiple linear regression analysis.

Results: We included 245 patients: 208 treated with allopurinol (66 on diuretics, 31.7\%), 35 with febuxostat (19 on diuretics, 57.6\%), and 2 with benzbromarone. Significantly fewer participants in the allopurinol plus diuretics subgroup achieved SU levels of less than $5 \mathrm{mg} / \mathrm{dL}$, but we found no other significant differences in SU targets associated with diuretics. Regarding the maximum ULD dose, a simple linear regression suggested an inverse relationship with diuretics (beta $=-0.125, p=0.073$ ), but this did not hold in the multivariable analysis (beta $=-0$. $47, p=0.833$ ). There was no association with febuxostat (beta $=-0.116, p=0.514$ ).
\end{abstract}

Conclusion: Diuretics do not appear to have a significant impact on managing gout.

Keywords: Gout, Diuretics, Furosemide, Thiazide, Serum urate, Allopurinol, Febuxostat

\section{Background}

Gout is the result of monosodium urate (MSU) crystal deposition in joints and periarticular tissues. It has become a major health issue with an increasing prevalence, affecting $0.7 \%$ to $3.7 \%$ of the population worldwide and as many as $5.9 \%$ of men in the USA [1]. It is the most common form of arthritis in western countries and is associated with significant morbidity [2], mortality [3] and health system costs [4]. Fortunately, proper management can stop flare-ups and achieve crystal dissolution and cure [5].

Hyperuricaemia, or the increase in serum urate (SU) levels, is a necessary condition for the development of

\footnotetext{
* Correspondence: drmarianoandres@gmail.com

${ }^{1}$ Rheumatology Deparment, Hospital General Universitario de

Alicante-ISABIAL, Pintor Baeza 12, 03010 Alicante, Spain

Universidad Miguel Hernández, Elche, Alicante, Spain

Full list of author information is available at the end of the article
}

gout. In the kidney, SU is easily filtered through the glomeruli and undergoes active reabsorption and secretion in the proximal convoluted tubule, with around $8 \%$ to $10 \%$ of the initially filtered SU being excreted [6]. Thus, disorders that impair the glomerular filtration of uric acid (such as renal failure) or interfere with its tubular transport (such as insulin-resistant states or drugs) would contribute to the development of hyperuricaemia and gout. As a result of this link, renal failure and diuretics are commonly seen in patients with gout [7].

Chronic kidney disease (CKD) has an impact on gout management by limiting the dosage or hampering the use of urate-lowering drugs (ULD), colchicine, and nonsteroidal anti-inflammatory agents [8]. The frequent prescription of diuretics might also impact outcomes in these patients [9]. Diuretics (both loop agents and thiazides) can increase SU levels but also interfere with 
allopurinol, enhancing the renal clearance of its active metabolite oxypurinol and increasing the dose needed to ensure effectiveness [10]. This would also apply to advanced heart failure or hypertension, where diuretics are often used. Febuxostat is used even in patients with advanced CKD based on its hepatic metabolism, though whether diuretics modify its urate-lowering effect is unknown. If not, its use would be preferential in patients with CKD or heart failure who are on diuretics, especially when diuretics cannot be discontinued.

In this context, there is a need to determine the impact of diuretics on gout management in clinical practice, especially after the incorporation of new ULD and treat-to-target strategies [11]. This knowledge could inform management plans for patients with gout who are taking diuretics, helping to achieve suitable SU reductions that ensure the cure of the disease. The aim of the present study was to assess the impact of diuretic therapy on the response to ULD in patients with gout, according to the type of drug prescribed.

\section{Methods}

We performed a retrospective analysis on an inception cohort in the Rheumatology Unit of the Hospital General Universitario de Alicante (Spain). The characteristics of the cohort have been described elsewhere [12]. Briefly, recruited patients are those presenting to the unit for the first time with crystal-proven gout without having received previous specialised care. The ethics committee of the Hospital General Universitario de Alicante (2017/05 Act) and the Spanish drugs regulatory administration approved this study; according to these organisations, informed consent was not necessary due to the retrospective nature of the study.

The study period for the present work was January 2014 to July 2017. All enrolled patients in the cohort were eligible for the analysis, which was based on a review of patients' electronic clinical and pharmacy records. We excluded patients without SU results during follow up.

\section{Study variables}

Our primary outcome variable was the maximum dose of ULD prescribed, as we considered this the best indicator of the potential influence of covariables - such as diuretics - in achieving proper SU reduction and the recommended SU targets [5] for managing patients with gout. Other outcome variables were (a) SU, both at baseline and under ULD at its maximum dose; (b) SU reduction achieved; and (c) the achievement of different SU targets $(6 \mathrm{mg} / \mathrm{dL} ; 5 \mathrm{mg} / \mathrm{dL} ; 4 \mathrm{mg} / \mathrm{dL})$.

The primary explanatory variable was the use and dosage of diuretic therapy, specifically loop agents (furosemide, torasemide) and thiazides, both at baseline and after achieving maximal dose of ULD. We also recorded the reason for prescribing diuretics (hypertension, heart failure, CKD, others, or mixed). Other explanatory variables were demographic (age, sex); clinical (hypertension, diabetes mellitus, dyslipidaemia, smoking, cardiovascular disease); anthropometric data (weight, height, body mass index (BMI)); laboratory measurements (estimated glomerular filtration rate (eGFR), according to the Chronic Kidney Disease Epidemiology Collaboration (CKD-EPI) formula [13]) at baseline and after maximal dose of ULD; and goutrelated data (years since first attack, numbers of attacks suffered, number of involved joints, joint pattern at presentation (monoarticular, oligoarticular, polyarticular), tophi, and type of ULD employed and prior usage).

\section{Statistical analysis}

We performed a descriptive analysis of the sample, expressing continuous variables as means (standard deviation (SD)) or medians (interquartile range ([IQR)), depending on the data distribution, and we expressed qualitative variables as frequencies and percentages. Based on clinical experience, we deemed a unified analysis of the use of diuretics inappropriate, as the patients eligible in clinics for certain ULDs (allopurinol, febuxostat, benzbromarone) are usually very heterogeneous, as are their pharmacodynamics and kinetics. To justify this division of the study population into subgroups based on the type of ULD prescribed, we compared explanatory variables using Student's $t$ test, Fisher's exact test, and the chi-squared test.

After that, we used Student's $t$ test, the Mann-Whitney $\mathrm{U}$ test, and the chi-squared test to assess the association between the primary explanatory variable (use of diuretics) and the different outcome variables, stratifying analyses by type of ULD. We considered that a patient was on diuretics when being treated at the moment of maximal dosage of ULD for at least a week, to avoid bias due to diuretic start or discontinuation during follow up. Manifest variability in the type and dosage of diuretics precluded subgroup analyses. In the case of significant results in the univariable analysis, we built a linear regression model for the primary outcome (maximal dose of ULD prescribed) to adjust for potential confounders (age, sex, hypertension, diabetes mellitus, dyslipidaemia, tobacco use, cardiovascular disease, BMI, baseline SU levels and eGFR, and final eGFR). All statistical analyses were performed using SPSS 22.0 software (IBM Statistics, Armonk, NY). Statistical significance was established at $p<0.05$.

\section{Results}

Of 296 patients enrolled in the inception cohort, followup data were available for 245 patients $(82.8 \%)$ with a median follow up of 8 months (IQR 4-13). General 
Table 1 Baseline characteristics of enrolled patients and group comparison according to the type of ULD used

\begin{tabular}{|c|c|c|c|c|}
\hline & Whole sample $(n=245)$ & Allopurinol $(n=208)$ & Febuxostat $(n=35)$ & $P$ \\
\hline Age (years), mean (SD) & $63.5(13.5)$ & $62.1(13.2)$ & $72.2(11.8)$ & $<0.001$ \\
\hline Male & $212(86.5)$ & $189(90.9)$ & $22(62.9)$ & $<0.001$ \\
\hline HT & $169(69.0)$ & $137(65.9)$ & $30(85.7)$ & 0.019 \\
\hline DM & $60(24.5)$ & $47(22.6)$ & $13(37.1)$ & 0.065 \\
\hline $\mathrm{DL}$ & $128(52.2)$ & $107(51.4)$ & $20(57.1)$ & 0.532 \\
\hline Smoking background $(n=234)$ & $140(59.9)$ & $128(64.3)$ & $12(36.4)$ & 0.006 \\
\hline CVD & $61(24.9)$ & $45(21.6)$ & $16(45.7)$ & 0.002 \\
\hline Diuretic therapy & $109(44.5)$ & $86(41.3)$ & $23(65.7)$ & 0.007 \\
\hline Type of diuretics $(n=243)$ & & & & 0.005 \\
\hline Thiazides & $61(25.1)$ & $51(24.5)$ & $10(28.6)$ & \\
\hline Loop agents & $46(18.9)$ & $33(15.9)$ & $13(37.1)$ & \\
\hline Combination of diuretics & $11(4.5)$ & $7(3.4)$ & $4(11.4)$ & 0.057 \\
\hline BMI $\left(\mathrm{kg} / \mathrm{m}^{2}\right)$, mean (SD) & $29.9(5.2)$ & $30.0(5.0)$ & $30.2(6.0)$ & 0.790 \\
\hline eGFR (mL/min), mean (SD) $(n=243)$ & $71.5(26.4)$ & $76.6(23.4)$ & $40.8(23.0)$ & $<0.001$ \\
\hline Chronic kidney disease $(n=241)$ & $76(31.5)$ & $47(22.8)$ & $29(82.9)$ & $<0.001$ \\
\hline $\mathrm{SU}(\mathrm{mg} / \mathrm{dL})$, mean $(\mathrm{SD})$ & $8.3(1.7)$ & $8.2(1.4)$ & $9.3(2.4)$ & 0.008 \\
\hline Prior use of ULD $(n=243)$ & $58(23.9)$ & $50(24.1)$ & $8(22.9)$ & 0.914 \\
\hline
\end{tabular}

Data shown as number (percentage) unless otherwise specified. In bold, statistically significant differences between allopurinol and febuxostat groups. BMI body mass index, calculated as weight: (height) ${ }^{2}, C V D$ cardiovascular disease, $D L$ dyslipidaemia,

DM diabetes mellitus, HT hypertension, SD standard deviation, SU serum urate, ULD urate-lowering drugs

baseline characteristics are shown in Table 1; these are typical of patients with gout seen in specialised clinical practice. Median disease duration at enrolment was 4 years (IQR 0-10), with a median of 4 reported flares (IQR 2-10) having involved a median of 3 joints (IQR 2.0-4.8). At presentation, 75.1\% of patients suffered from a monoarticular flare, $19.2 \%$ from an oligoarticular flare, and $5.3 \%$ from a polyarticular flare. Tophi were recorded in $23.3 \%$ of patients.

At baseline, 109 patients (44.5\%) were on diuretics: $63.0 \%$ of these for an indication of hypertension, $12.6 \%$ for heart failure, $5.0 \%$ for CKD, and $19.3 \%$ for combined/other causes. More patients were taking thiazides than loop agents (57.0\% versus $43.0 \%)$, and 17 (5.7\%) patients were taking both. When the maximal dose of ULD was reached, 90 patients were on diuretics (36.7\%); these had been stopped in 25 cases (22.9\%) and started in 6 (4.4\%). At this time point, more patients were taking loop diuretics than thiazides $(55.5 \%$ versus $44.6 \%) ; 8$ (3.3\%) were on combined diuretic therapy. The supplementary material describes dosage at both time points (Additional file 1: Table S1).

With regard to ULDs, 208 patients (84.9\%) were on allopurinol; 35 (14.3\%) were on febuxostat; and 2 (0.8\%) were on benzbromarone (excluded from the analysis). Table 1 compares baseline characteristics according to ULD treatment: the febuxostat group was significantly older, included more women, and had a higher prevalence of hypertension, CKD, and cardiovascular disease.
On the other hand, there were fewer smokers, and their blood tests revealed higher levels of SU and lower eGFR. Given the significant heterogeneity, we did not pool groups but report results separately according to type of ULD.

\section{Patient outcomes: allopurinol group}

Table 2 shows the outcomes in the 208 patients receiving allopurinol ( $n=66$, or $32.7 \%$ also on diuretics). The most frequently prescribed diuretic was hydrochlorothiazide $(n=37,56.0 \%)$ at either $12.5 \mathrm{mg} /$ day $(n=19)$ or 25 $\mathrm{mg} /$ day $(n=13) ; 8$ patients $(12.1 \%)$ were on combined diuretic therapy. Despite higher final SU levels in those taking diuretics compared to those who were not, the average SU reduction was similar between subgroups. We did not observe significant differences in the achievement of the different SU targets, except for $\mathrm{SU}$ of less than $5 \mathrm{mg} / \mathrm{dL}$, with lower rates in the diuretic subgroup.

The maximum doses of allopurinol were comparable between subgroups, though they tended to be lower in those on diuretics and especially in those on diuretic combination; there was no difference between thiazides and loop agents (Additional file 1: Table S2). The simple linear regression analysis also suggested inverse correlation between maximum dose of allopurinol and use of diuretics (beta $=-0.125,95 \%$ CI -67.7 to 3.0, $p=0.073)$. However, this association was lost after 
Table 2 Outcomes of the allopurinol group and comparisons on the use of diuretics

\begin{tabular}{|c|c|c|c|}
\hline & $\begin{array}{l}\text { No diuretics } \\
(n=142(68.3 \%))\end{array}$ & $\begin{array}{l}\text { Diuretics } \\
(n=66(31.7 \%))\end{array}$ & $P$ \\
\hline \multicolumn{4}{|l|}{ Primary outcome variable } \\
\hline $\begin{array}{l}\text { Maximum dose of } \\
\text { allopurinol (mg/day) }\end{array}$ & 304.9 (120.6) & $275.8(119.7)$ & 0.109 \\
\hline \multicolumn{4}{|l|}{ Secondary outcome variables } \\
\hline Baseline SU (mg/dL) & $8.1(1.4)$ & $8.4(1.6)$ & 0.116 \\
\hline Final SU (mg/dL) & $4.9(1.3)$ & $5.4(1.7)$ & 0.015 \\
\hline Final eGFR (mL/min) & $83.0(24.4)$ & $64.6(21.5)$ & $<0.001$ \\
\hline SU reduction (mg/dL) & $3.2(1.8)$ & $3.0(1.9)$ & 0.474 \\
\hline \multicolumn{4}{|l|}{ Achievement of SU targets } \\
\hline - SU <6 mg/dL, n (\%) & $110(79.1)$ & $47(68.1)$ & 0.082 \\
\hline - SU $<5$ mg/dL, n (\%) & $75(54.0)$ & $27(39.1)$ & 0.044 \\
\hline - SU $<4$ mg/dL, n (\%) & $34(24.5)$ & $11(15.9)$ & 0.160 \\
\hline
\end{tabular}

Data shown as mean (SD) unless specified otherwise. In bold, statistically significant differences between subgroups. eGFR estimated glomerular filtration rate, $S D$ standard deviation, $S U$ serum urate

adjusting for covariates (beta $=-4.70,95 \% \mathrm{CI}-48.3$ to $38.9, p=0.833)$.

\section{Patient outcomes: febuxostat group}

Table 3 shows the results of patients on febuxostat. Of the 33 patients, 19 (57.6\%) were on diuretics. Here, the use of furosemide predominated $(n=13(68.4 \%))$ at doses of $40 \mathrm{mg} /$ day $(n=5), 60 \mathrm{mg} /$ day $(\mathrm{n}=1), 80 \mathrm{mg} /$ day $(n=2)$, and $120 \mathrm{mg} /$ day $(n=3)$. Two patients $(10.5 \%)$ were on combination therapy. We did not observe significant differences in the reduction of SU levels or the achievement of the different SU targets.

Table 3 Outcomes of the febuxostat group and comparisons on the use of diuretics

\begin{tabular}{|c|c|c|c|}
\hline & $\begin{array}{l}\text { No diuretics } \\
(n=14(42.4 \%))\end{array}$ & $\begin{array}{l}\text { Diuretics } \\
(n=19(57.6 \%))\end{array}$ & $P$ \\
\hline \multicolumn{4}{|l|}{ Primary outcome variable } \\
\hline $\begin{array}{l}\text { Maximum dose of } \\
\text { febuxostat (mg/day) }\end{array}$ & $80.0(70.0-80.0)$ & $80.0(40.0-80.0)$ & 0.398 \\
\hline \multicolumn{4}{|l|}{ Secondary outcome variables } \\
\hline Baseline SU (mg/dL) & $8.7(7.6-9.6)$ & $9.6(8.0-11.4)$ & 0.174 \\
\hline Final SU (mg/dL) & $3.5(2.5-4.9)$ & $3.9(3.4-5.1)$ & 0.439 \\
\hline Final eGFR (ml/min) & $49.1(37.3-69.1)$ & $30.5(19.0-42-9)$ & 0.004 \\
\hline $\mathrm{SU}$ reduction $(\mathrm{mg} / \mathrm{dL})$ & $5.0(2.5-5.6)$ & $4.5(3.9-6.3)$ & 0.733 \\
\hline \multicolumn{4}{|c|}{ Achievement of SU targets } \\
\hline - SU $<6$ mg/dl, n (\%) & 11 (78.6) & $17(81.0)$ & 1.000 \\
\hline - SU $<5$ mg/dl, n (\%) & $11(78.6)$ & $14(66.7)$ & 0.445 \\
\hline - SU $<4$ mg/dl, n (\%) & $8(57.1)$ & $11(52.4)$ & 0.782 \\
\hline
\end{tabular}

Data shown as median (IQR) unless specified otherwise. In bold, statistically significant differences between subgroups. eGFR estimated glomerular filtration rate, SU serum urate
The average maximum dose of febuxostat was $80 \mathrm{mg} /$ day in both subgroups, with no association with the type of diuretic (Additional file 1: Table S2). The simple regression analysis showed no correlation (beta $=-0.116,95 \%$ CI - 23.7, 12.1, $p=0.514$ ), so further adjustment was unnecessary.

\section{Discussion}

The present study assessed the impact of diuretics in managing gout in an inception cohort of patients in specialised care. This issue is a subject of debate in the literature, with conflicting data in the case of allopurinol [10, 14], but we did not observe any significant effect of diuretics on the achievement of SU targets or on the maximum doses prescribed in patients on allopurinol or febuxostat.

Diuretics, especially loop agents and thiazides, are extensively used in clinical practice and are often essential for managing potentially life-threating disorders such as heart or kidney failure. Diuretic-induced hyperuricaemia is a well-established secondary effect and derives from impaired SU excretion due to reducing extracellular volume [15] and interactions with renal urate transport at the proximal convoluted tubule [16]. Diuretics are common in patients with gout, who tend to be older and suffer from a number of comorbidities [17]. In the present study, more than $40 \%$ of the study population was on diuretics at baseline. However, whether the development of gout relates to the diuretic-induced hyperuricaemia or is attributable to the underlying disease is still unclear [18].

The dosage of allopurinol needed to achieve SU targets varies widely between patients, depending on variables such as kidney function, body size, or genetic factors influencing tubular reabsorption [19]. Concomitant use of diuretics was shown to increase the doses of allopurinol necessary to reach certain SU levels by $25 \%$ to $100 \%$ [10]. However, in our adjusted analysis there were no differences in allopurinol dosage based on concomitant diuretic therapy. Wright et al. analysed data from five clinical trials, while here patients were recruited in a clinical practice setting, which is often more complex due to the prevalence of comorbidities. Loop diuretics and thiazides were analysed together in both studies, with no stratification according to dosage in the present study due to the risk of an underpowered analysis. For furosemide, some authors have suggested that allopurinol interferes with down-regulation of xanthine oxidase, explaining higher dosage requirements [20], but this hypothesis has not been fully confirmed [21]. In contrast, no authors have observed significant interactions with thiazides [22, 23]. Further studies should assess the existence of a differential effect in allopurinol dosage between types of diuretics.

Regarding the impact of diuretics on the effect of febuxostat, hydrochlorothiazide showed no significant 
clinical interaction [24], and we did not identify any studies assessing loop agents from the current literature. The present study showed no differences in dosage or achievement of SU targets based on the use of diuretics. These findings - the first to come from clinical practice would lend support to the use of febuxostat in this setting. However, they should be interpreted with caution, as our sample included few participants taking this drug, and moreover, febuxostat is only marketed in Europe as $80 \mathrm{mg}$ and $120 \mathrm{mg}$ tablets (thus limiting its dosage, unlike allopurinol). Larger studies are needed to corroborate our results.

Almost a quarter of the patients in the study sample had their diuretics discontinued during follow up. Although this treatment strategy is in line with recommendations from the European League Against Rheumatism (EULAR) [5], these were based on low-quality evidence, and our results do not support this course of action, at least in the case of urate-lowering therapy. Nevertheless, the approach may be of interest when the patient reaches persistent normouricaemia and there is a clinical presumption of complete removal of crystal deposits. ULD dose reduction or discontinuation should aim to maintain uricaemia below its saturation point to prevent new crystal formation [25]. At this point, the absence of factors contributing to hyperuricaemia, such as diuretics, is desirable. However, this research question, while of active interest, was beyond the scope of the present study.

The main strength of this study is its structured assessment of outcomes in a cohort of patients with crystal-proven gout. We analysed a significant sample size of more than 200 patients treated with allopurinol, compared to 133 patients included in the study by Wright et al. [9], allowing a proper evaluation of outcomes in the allopurinol group. Furthermore, we considered several covariates, adjusting the analysis to reinforce the robustness of the results. Regarding the limitations, these mainly relate to the cohort study design and include the absence of random sampling and allocation to ULDs or diuretics, along with the impossibility of modifications to the treatment regimens by researchers. Around 20\% of patients included in the inception cohort were excluded from this analysis due to loss of follow up from clinics, a phenomenon that often occurs in clinical practice [26]; however, we analysed complete data in around 250 patients, limiting the impact of these losses. We did not undertake a differential analysis on the type of diuretic, owing to small numbers in the subgroups. Although the results are comparable with current evidence, this should be properly assessed in the future. The effect of diuretics on the duration of achieving the recommended SU targets could provide an additional idea of their impact on management; unfortunately, this issue could not be assessed in the present study as records review focused on the time of maximum dose of ULD prescribed - the primary study outcome. We did not assess safety.

\section{Conclusion}

Both loop agents and thiazides are diuretics that are commonly prescribed in patients with gout and have often been associated with impaired response to allopurinol and refractoriness, prompting recommendations for their discontinuation when possible. However, the results of the present study do not support prior observations related to the achievement of SU targets or the necessary ULD dosage, while they are in keeping with those recently reported by Kannangara et al. [14]. These findings require further confirmation through prospective, intervention studies.

\section{Additional file}

Additional file 1: Table S1. Type and dosage of diuretics. Table S2. Comparison of maximum dose of urate-lowering drugs regarding the type of diuretics. (DOCX $15 \mathrm{~kb}$ )

\section{Abbreviations}

BMI: Body-mass index; CKD: Chronic kidney disease; CKD-EPI: Chronic kidney disease epidemiology collaboration; IQR: Interquartile range; MSU: Monosodium urate; SD: Standard deviation; SU: Serum urate; ULD: Urate-lowering drugs

\section{Acknowledgements}

The authors are grateful to Prof. Eliseo Pascual for his suggestions on the manuscript and to Mrs. Meggan Harris for the English revision.

\section{Funding}

The present work was funded by a grant from the Institute of Health and Biomedical Research of Alicante-ISABIAL (file: UGP-16-156).

\section{Availability of data and materials}

Please contact author for data request.

Authors' contributions

$L R, C C, P Z$, and MA conceptualized and designed the study. $L R, C C, M L P$, and IC retrieved the data. $L R, C C, P Z$, and $M A$ analysed the data and interpreted the results. LR, CC and MA wrote the first draft of the manuscript. All authors read and approved the final version of the manuscript.

\section{Ethics approval and consent to participate}

The ethics committee of the Hospital General Universitario de Alicante (2017/05 act) and the Spanish drugs regulatory administration approved this study; according to these organisations, informed consent was not necessary due to the retrospective nature of the study.

Consent for publication

Not applicable.

\section{Competing interests}

MA has received speaking and advisory fees from Menarini, Astra-Zeneca, Horizon, and Grünenthal. The other authors declare no conflicts of interest in the conduct of the present study.

\section{Publisher's Note}

Springer Nature remains neutral with regard to jurisdictional claims in published maps and institutional affiliations. 


\section{Author details}

'Rheumatology Deparment, Hospital General Universitario de Alicante-ISABIAL, Pintor Baeza 12, 03010 Alicante, Spain. ${ }^{2}$ Universidad Miguel Hernández, Elche, Alicante, Spain. ${ }^{3}$ Clinical Pharmacology Deparment, Hospital General Universitario de Alicante-ISABIAL, Alicante, Spain.

Received: 26 October 2017 Accepted: 6 March 2018

Published online: 22 March 2018

\section{References}

1. Roddy E, Choi HK. Epidemiology of gout. Rheum Dis Clin N Am. 2014;40:155-75.

2. Scire CA, Manara M, Cimmino MA, et al. Gout impacts on function and health-related quality of life beyond associated risk factors and medical conditions: results from the KING observational study of the Italian Society for Rheumatology (SIR). Arthritis Res Ther. 2013;15:R101.

3. Clarson LE, Chandrate P, Hider SL, et al. Increased cardiovascular mortality associated with gout: a systematic review and meta-analysis. Eur J Prev Cardiol. 2015;22:335-43.

4. Jackson R, Shiozawa A, Buysman EK, Altan A, Korrer S, Choi H. Flare frequency, healthcare resource utilisation and costs among patients with gout in a managed care setting: a retrospective medical claims-based analysis. BMJ Open. 2015;5:e007214.

5. Richette P, Doherty M, Pascual E, et al. 2016 updated EULAR evidence-based recommendations for the management of gout. Ann Rheum Dis. 2017;76:29-42.

6. Pascual E, Perdiguero M. Gout, diuretics and the kidney. Ann Rheum Dis. 2006:65:981-2

7. Richette P, Clerson P, Perissin L, Flipo RM, Bardin T. Revisiting comorbidities in gout: a cluster analysis. Ann Rheum Dis. 2015:74:142-7.

8. Vargas-Santos AB, Neogi T. Management of gout and hyperuricemia in CKD. Am J Kidney Dis. 2017;70(3):422-39.

9. Bardin T, Richette P. Impact of comorbidities on gout and hyperuricaemia: an update on prevalence and treatment options. BMC Med. 2017;15:123.

10. Wright DF, Duffull SB, Merriman TR, Dalbeth N, Barclay ML, Stamp LK. Predicting allopurinol response in patients with gout. Br J Clin Pharmacol. 2016;81:277-89

11. Kiltz U, Smolen J, Bardin T. Treat-to-target (T2T) recommendations for gout. Ann Rheum Dis. 2017;76:632-8

12. Andrés M, Bernal JA, Quilis N, Sivera F, Carmona L, Vela P, Pascual E. Cardiovascular risk of gout patients seen at rheumatology clinics following a structured assessment. Ann Rheum Dis. 2017;76:1263-8.

13. Levey AS, Stevens LA, Schmid CH, Zhang YL, Castro AF 3rd, Feldman HI, et al. A new equation to estimate glomerular filtration rate. Ann Int Med. 2009; 150:604-12.

14. Kannangara DRW, Graham GG, Wright DFB, et al. Individualising the dose of allopurinol in patients with gout. Br J Clin Pharmacol. 2017;83:2015-26.

15. Weinman EJ, Eknoyan G, Suki WN. The influence of the extracellular fluid volume on the tubular reabsorption of uric acid. J Clin Invest. 1975:55:283.

16. McAdams-DeMarco MA, Maynard JW, Baer AN, et al. A urate gene-bydiuretic interaction and gout risk in participants with hypertension: results from the ARIC study. Ann Rheum Dis. 2013;72:701-6.

17. Mitnala S, Phipps-Green A, Franklin C, et al. Clinical and genetic features of diuretic-associated gout: a case-control study. Rheumatology (Oxford). 2016;55:1172-6.

18. Janssens HJEM, van de Lisdonk EH, Janssen M, van den Hoogen HJM, Verbeek ALM. Gout, not induced by diuretics? A case-control study from primary care. Ann Rheum Dis. 2006;65:1080-3.

19. Stamp LK, Merriman TR, Barclay ML, et al. Impaired response or insufficient dosage? Examining the potential causes of "inadequate response" to allopurinol in the treatment of gout. Semin Arthritis Rheum. 2014;44:170-4

20. Knake C, Stamp L, Bahn A. Molecular mechanism of an adverse drug-drug interaction of allopurinol and furosemide in gout treatment. Biochem Biophys Res Commun. 2014;452:157-62.

21. Stamp LK, Barclay ML, O'Donnell JL, et al. Furosemide increases plasma oxypurinol without lowering serum urate-a complex drug interaction: implications for clinical practice. Rheumatology (Oxford). 2012;51:1670-6.

22. Hande $K$, et al. Evaluation of a thiazide-allopurinol drug interaction. Am J Med Sci. 1986;292:213-6.
23. Loffler W, Landthaler R, de Vries J, et al. Interaction of allopurinol and hydrochlorothiazide during prolonged oral administration of both drugs in normal subjects. Clin Investig. 1994;72:1071-5.

24. Grabowski B, Khosravan R, Wu JT, et al. Effect of hydrochlorothiazide on the pharmacokinetics and pharmacodynamics of febuxostat, a non-purine selective inhibitor of xanthine oxidase. Br J Clin Pharmacol. 2010;70:57-64.

25. Perez-Ruiz F, Herrero-Beites AM, Carmona L. A two-stage approach to the treatment of hyperuricemia in gout: the "dirty dish" hypothesis. Arthritis Rheum. 2011:63:4002-6.

26. Darmawan J, Rasker JJ, Nuralim H. The effect of control and self-medication of chronic gout in a developing country. Outcome after 10 years. J Rheumatol. 2003;30:2437-43.

\section{Submit your next manuscript to BioMed Central and we will help you at every step:}

- We accept pre-submission inquiries

- Our selector tool helps you to find the most relevant journal

- We provide round the clock customer support

- Convenient online submission

- Thorough peer review

- Inclusion in PubMed and all major indexing services

- Maximum visibility for your research

Submit your manuscript at www.biomedcentral.com/submit 